Sharpe, M. E. (1955). J. gen. Microbiol. 13, 198-199.

\title{
Haptene Substances in Culture Media for Lactobacilli
}

\author{
By M. ELISABETH SHARPE \\ National Institute for Research in Dairying, University of Reading
}

SUMMARY: Tomato juice Yeastrel Tween 80 broth was found to contain two haptene substances, one a component of tomato juice and the other of Yeastrel. These caused difficulties in the use of lactobacillus antisera. Methods were devised for eliminating the effects of these haptenes.

Non-antigenic constituents of bacteriological media have been found on occasion to stimulate the production of antibodies when organisms grown in the media were inoculated into animals, presumably owing to adsorption of the substances onto the bacterial cells (e.g. Sordelli \& Mayer, 1931 $a, b$; Morgan, 1936; Glynn \& Holborow, 1952). To produce antisera for work on the serology of lactobacilli (Sharpe, 1955) strains of lactobacilli for inoculation into rabbits were grown in the tomato juice Yeastrel Tween 80 broth (TDB) described by Briggs (1953). The antisera obtained from rabbits after several courses of inoculation with lactobacilli were found to react by precipitin test with $\mathrm{HCl}$ extracts of all the species of lactobacilli tested, completely obscuring the specific reactions. Sterile TDB medium also reacted with these nonspecific antisera and tests on the different components of this medium showed that only the tomato juice gave a positive reaction. The non-specificity was thus due to a constituent of tomato juice which was adsorbed on to lactobacilli grown in the medium; then when these were used as antigen, antibodies for tomato juice were produced. Such sera reacted with the $\mathbf{H C l}$ extracts, as organisms for extraction were also grown in TDB medium. When rabbits were inoculated over a period of several months with tomato juice or TDB medium alone no antibodies for tomato juice were detected in the sera, indicating that the material in the tomato juice was a haptene and not a complete antigen. When lactobacilli for inoculation into rabbits were grown in TDB medium without Tween 80, the production of antibodies for tomato juice was delayed but eventually they appeared. Growth of some strains of lactobacilli was very poor in this medium. Absorption of tomato juice antisera with organisms of any heterologous strain of lactobacilli grown in TDB medium removed the tomato juice antibodies from the serum but also sometimes removed antibodies specific for lactobacilli (Sharpe, 1955).

Dialysis of tomato juice against distilled water showed that the haptene did not dialyse through a cellophan membrane. However, a medium of similar composition to TDB medium but prepared with the outer solution which had passed through the membrane gave a sparser growth, equivalent to the growth in TDB medium without tomato juice. Growth-promoting substances had evidently also been retained by the membrane, or so diluted as to be ineffective. Attempts to free lactobacilli grown in TDB medium from the tomato haptene before preparing $\mathrm{HCl}$ extracts were unsuccessful, neither could it be separated from these extracts by ethanol. As the removal of tomato juice antibodies from the sera or of the tomato juice antigen from $\mathrm{HCl}$ extracts of organisms 
proved impractical, the difficulty was overcome by growing lactobacilli for rabbit inoculation in a modified TDB medium, namely, without tomato juice, and using the complete TDB medium for growing organisms to be extracted for antigen for in vitro tests. The tomato juice antigen in the extracts was thus rendered ineffective, since the sera did not contain haptene antibodies. With this method, however, non-specific antibodies again appeared in the sera, although the reaction was less potent than previously, and it took longer for such antibodies to appear. This effect was traced to the Yeastrel, used in compounding the TDB medium, which gave rise to Yeastrel antibodies. This reaction was eliminated by omitting Yeastrel from the TDB medium used to grow the organisms to be used for preparing extracts.

Subsequently therefore as a routine, the effects of these haptenes were eliminated as follows: lactobacilli for rabbit inoculation were grown in a medium containing Yeastrel but no tomato juice and lactobacilli for preparing antigen extracts in a medium containing tomato juice but no Yeastrel. Since tomato juice and yeast extracts are increasingly used for culture media similar difficulties might arise in serological work with other organisms and might be avoided in the same way.

Lactobacilli grown in TDB medium appear readily to adsorb haptenes from the medium, and the fact that the presence of tomato juice antibodies in sera was very much delayed by the absence of Tween 80 from the medium suggests that this substance may have had some effect on the adsorption. In this connexion it is of interest that Middlebrook \& Dubos (1947) found that tubercle bacilli grown in a medium containing Tween 80 adsorbed the Tween and inoculation of these organisms produced anti-Tween sera which reacted with Tween 80 by precipitin test and agglutinated tubercle bacilli grown in the medium. Contrary to these findings anti-Tween sera were not demonstrated with the lactobacilli used here: no antisera precipitated with Tween 80 or agglutinated organisms grown in the Tween containing TDB medium.

I wish to thank Dr A. T. R. Mattick for his interest in this work, and Dr N. J. Berridge for helpful discussions.

\section{REFERENCES}

Briggs, M. (1953). An improved medium for lactobacilli. J. Dairy Res. 20, 36.

Glynn, L. E. \& Holborow, E. J. (1952). The production of complete antigens from polysaccharide haptenes by streptococci and other organisms. J. Path. Bact. 64, 775 .

Middlebrook, G. \& Dubos, R. J. (1947). The effect of tubercle bacilli on the antigenicity of a synthetic ester of oleic acid. J. Immunol. 56, 301.

Morgan, W. T. J. (1936). Studies in immunochemistry. The preparation and properties of a specific polysaccharide from B. dysenteriae Shiga. Biochem. J. 30, 909.

Sharpe, M. E. (1955). A serological classification of lactobacilli. J. gen. Microbiol. $12,107$.

Sordellt, A. \& Mayer, E. (1931 a). Les précipitines de la gélose. C.R. Soc. Biol., Paris, 107, 736.

Sordelli, A. \& Mayer, E. (1931 b). L'Agar comme antigène in vitro. C.R. Soc. Biol., Paris, 108, 675. 